\title{
Roughness perception across the hands
}

\author{
Roberta D. Roberts
}

Published online: 8 May 2013

(C) Psychonomic Society, Inc. 2013

\begin{abstract}
It has previously been shown that the perceived roughness of a surface touched by one digit is influenced by the roughness of a different surface touched simultaneously by another digit on the same hand. The present study was designed to examine whether this is the case when surfaces of varying roughness are touched using digits on separate hands. Participants touched pairs of sandpaper surfaces, in sequence, using the same digit, and identified which of the two was rougher. Roughness discrimination was measured in the presence of distractor surfaces touched simultaneously with the target surface, but using a different digit either on the same or on the other hand. The overall perception of roughness of the attended surfaces was better on the left than on the right hand. Perceived roughness also varied systematically with the roughness of the distractor surfaces. Attended surfaces were more likely to be perceived as smoother when they were paired with smooth rather than rough distractors. Likewise, attended surfaces tended to be perceived as rougher with rough distractors. This pattern of results occurred whether the attended and distractor digits were on the same hand or different hands. These data confirm that it is difficult to restrict tactile attention for roughness to a single digit and show that this difficulty extends to restricting attention to a single hand. Furthermore, the effect of a stimulus at an unattended body location was not simply to impair perception in general, but to bias it in the roughness direction of the distractor surface.
\end{abstract}

Keywords Selective attention $\cdot$ Texture $\cdot$ Touch

Feeling the texture of an object seldom involves the use of only a single finger. Rather, in unconstrained settings, the textures of objects are actively explored using sweeps of multiple digits. If these back-and-forth tangential movements across the object surfaces (Lederman \& Klatzky, 1987) are

R. D. Roberts $(\square)$

School of Psychology, University of Birmingham, Edgbaston,

Birmingham B15 2TT, UK

e-mail: R.Roberts@bham.ac.uk made by moving the hand, the result is highly coupled information across the digits. Despite this, little is known at present about whether and how the brain might combine this information from multiple contact points in order to generate an overall sense of the texture of touched surfaces.

When the whole hand is used to feel an object's texture, it is possible that the signals from each digit may be processed independently. The resulting sensation for a digit may then be identified irrespective of the sensory inputs from other digits on the same or different hands. Alternatively, the signals from the digits may interact at various stages of somatosensory processing, making it difficult to isolate the sensation on one digit from those on others. Recent evidence has suggested that the perceived roughness of an actively explored surface may be the result of the combination of signals from multiple contacting digits. Using the method of absolute magnitude estimation, Verrillo and colleagues (Verrillo, Bolanowski, \& McGlone, 1999) measured roughness judgments for sandpaper stimuli presented to the index finger, thumb, or both digits. Although they found no difference in estimates of sandpaper roughness when using either the index finger or the thumb, significantly higher values (indicating greater perceived roughness) were assigned when both digits were used simultaneously. The authors interpreted this finding as evidence for a form of spatial summation over the two digits. Consistent with this interpretation, Roberts and Humphreys (2010a) and Kahrimanovic, Bergmann Tiest, and Kappers (2009) found that the surface roughness at a task-irrelevant and unattended (distractor) digit systematically influenced the perceived roughness at an attended (target) digit. Reports of the roughness at the target digit were biased toward smoother judgments when accompanied by a smooth texture on the distractor digit, and toward rougher judgments when accompanied by rough textures. Thus, when two digits on a hand simultaneously touched textured surfaces, it was difficult to restrict attention to the roughness experienced by just one of the digits.

Thus far, the majority of work examining roughness perception during multidigit contact has made use of pairs of digits from the same hand. The present study was 
designed to investigate whether interactions between concurrently stimulated digits would be observed when those digits belonged to different hands. Currently, the findings from studies of multidigit texture perception suggest that the interaction between texture signals would be restricted to digits from a single hand. For example, sensory interactions between concurrently stimulated digits have also been shown to be constrained by their relative separation on the body's surface. Roberts and Humphreys (2010a) found that the nature of the interaction between simultaneously stimulated digits changed when those digits occupied distant rather than adjacent locations on the hand. Whereas neighboring digits showed patterns of interaction consistent with the integration of roughness signals, stimuli applied to more somatotopically distant distractor digits decreased roughness sensitivity overall at the attended digit. Together with the results of Verrillo et al. (1999) and Kahrimanovic et al. (2009), the latter finding suggests that integration of roughness signals between the digits is most likely to occur in areas of the brain primarily activated by somatosensory inputs from one side of the body and whose afferent cells have receptive fields spanning neighboring digits. If the integration of sensory inputs signaling roughness does take place in such regions, there should be no interaction between roughness signals originating from widely separated sites on the body's surface. Therefore, a tactile stimulus applied to the digit of one hand should have a minimal effect on the perception of roughness at a digit on another hand.

To date, only one study has examined roughness perception using surfaces presented on multiple sites on different sides of the body. Dorsch, Hsiao, Johnson, and Yoshioka (2001) used subjective magnitude ratings to examine roughness perception during the active exploration of sandpaper surfaces. In agreement with previous work examining roughness perception using sandpaper surfaces (Ekman, Hosman, \& Lindstrom, 1965; Hollins \& Risner, 2000), they found that perceived roughness increased with surface roughness. That is, sandpaper surfaces with lower grit values, which have larger particles, were perceived as being rougher than those with higher grit values (smaller particles). However, in contrast to the work of Kahrimanovic et al. (2009) and Roberts and Humphreys (2010a), Dorsch et al. found no effect of distractor roughness when the concurrently stimulated target and distractor digits were on the same hand (i.e., the right index and middle fingers). Moreover, their work showed that the perceived roughness of a surface explored using the index finger on one hand was unaffected by the roughness of surfaces simultaneously touched by the other index finger. This absence of a stimulus interaction between the digits on different hands raises the possibility that it may be possible to restrict attention to the roughness signals from one side of the body, even to a single digit (contrary to the results of Kahrimanovic et al., 2009, and Roberts \& Humphreys, 2010a). However, the difference in findings from other studies on unilateral roughness perception is surprising. The discrepancy may be due to methodological differences, such as the use of subjective magnitude ratings by Dorsch and colleagues rather than the discrimination experimental designs used by Kahrimanovic et al. and Roberts and Humphreys (2010a). Furthermore, given findings that it is difficult to restrict tactile attention for vibrotactile stimuli to one side of the body (Driver \& Grossenbacher, 1996; Lakatos \& Shepard, 1997; Roberts \& Humphreys, 2010b; see the General Discussion below for further details), it is possible that the perception of roughness at a cued digit will be affected by the roughness of spatially close distractor stimuli, regardless of whether those distractors are on the same or on a different hand.

The two experiments described here were designed to investigate whether surface roughness at a distractor digit, previously found to influence the perception of surfaces touched by another digit on the same hand, would also influence the perception of surfaces touched by a digit on a different hand. Roughness discrimination was assessed in the presence of distractor surfaces on the same side and on different sides of the body. This was carried out using sandpaper surfaces, as well as a discrimination task and digit combinations previously shown by Roberts and Humphreys (2010a) and Verrillo et al. (1999) to exhibit interdigit interactions. The between-subjects design and measurement of digit sensory interactions across a large number of participants in Experiment 1 increased the ease with which the present results could be generalized to larger groups while minimizing possible learning effects. In Experiment 2, digit sensory interactions were then investigated using a within-subjects design, so that the increased experimental power raised the chances of finding differences between surface exploration with one and two hands. In Experiment 1, the role of the different digits in generating interference between the digits was also assessed by measuring performance with either the thumb or the index finger as the distractor digit. Similarly, the role of the hand to which the distractor digits belonged was investigated by measuring roughness discrimination with distractors touched by the left versus the right hand.

\section{Experiment 1}

\section{Method}

Participants Data were collected from 90 volunteers ( 80 women, ten men) between 16 and 34 (median 19) years of age. All of the participants were right-handed, on the basis of self-report, and all had normal or corrected-to-normal vision, gave informed consent, and received $£ 3$ sterling for 
their participation. The participants were randomly assigned to one of six experimental groups. Details of these groups can be found in Table 1 .

Stimuli Roughness perception was tested using stimuli composed of sandpaper textures with grit values of 80 (192), 180 (80), 240 (50), and 320 (40). Larger values indicate decreasing roughness, and the values in parentheses show mean particle sizes in micrometers. Each stimulus was made of four strips of sandpaper attached to the corner surfaces of one end of a piece of card (see Fig. 1 for pictures).

The experiment was made up of a series of 40 trials. In each trial, the participants used a thumb and index finger (combined into a pinch grip posture-see Fig. 2) to make sliding contact with a pair of sandpaper surfaces simultaneously. Each stimulus pair was composed of two sandpaper surfaces mounted on opposite sides of a piece of card (see Fig. 1a), with each surface being touched by one of the two digits. The participants were asked to attend to and judge the roughness of one of these sandpaper surfaces (the target surface) and to ignore the sensations arising from the sandpaper surface on the other side of the card (the distractor surface). A second target/distractor pair was then presented and the exploratory action repeated. The two target surfaces were compared, and participants reported which of the two targets was rougher. Thus, for each trial, the participant felt two target surfaces, one after the other using the same digit, and judged their relative roughness. The second digit, touching the distractor surfaces, could be on the same or on a different hand from the digit touching the target surfaces.

The 180- and 240-grit sandpapers were used as the pair of target surfaces on every trial. A pilot study with five participants indicated that this pairing of surfaces would lead to an error rate of between $10 \%$ and $20 \%$. This error rate was chosen as a compromise between ensuring that most participants were able to perform the task and generating errors in the different conditions. One of the target/distractor pairings always had the same level of grit for both surfaces (the standard stimulus). The other pairing had a target surface paired equally often with a distractor surface that was relatively rougher, equally rough, or smoother (the comparison stimuli). For 30 of the 40 trials in the experiment (regular trials), the smoother of the target surfaces (240 grit) was used in the standard stimulus, producing a target/distractor pairing of 240/240 grit. The rougher target (180 grit) was used as the target surface in the comparison stimulus and was paired equally often with a rougher ( 80 grit), an equally rough (180 grit), and a smoother (320 grit) distractor surface. Each of these three target/distractor pairings was touched ten times, making up the 30 trials. Therefore, the standard trials were pairings of the standard stimulus (240/240 grit) with ten of each of the following comparison stimulus pairs: 180/320 (smoother distractor), 180/180 (equal distractor), and 180/80 (rougher distractor) grit. For the remaining ten trials (rough-swapped trials), the relatively rougher target (180 grit) was used as the standard stimulus, and the smoother target (240 grit) was paired with the rougher ( 80 grit) distractor surface. Thus, for these ten trials, the standard stimulus pairing was $180 / 180$ grit and the comparison stimulus pairing was 240/80 grit.

In the regular trials, as in those of Roberts and Humphreys (2010a), the distractors (rough, smooth, or equal) were always presented with the rougher of the two target surfaces. Therefore, the presence of a rough or smooth distractor provided a potential additional cue to the temporal location of the rough target surface. Although smooth distractors were difficult to explicitly distinguish from the 180-grit target surface, the distinctive rough distractors were easy to tell apart from the rough target surface (see Roberts \& Humphreys, 2010a) and from all of the other textures (participants' subjective reports). Therefore, a possible strategy in trials containing rough distractors would have been to

Table 1 Experimental details of each group of participants

\begin{tabular}{|c|c|c|c|c|c|c|c|c|}
\hline \multirow[t]{2}{*}{ Group } & \multirow[t]{2}{*}{ Attended Digit } & \multirow[t]{2}{*}{ Distractor Digit } & \multirow[t]{2}{*}{ Total $N$} & \multirow[t]{2}{*}{ Excluded $N$} & \multicolumn{4}{|c|}{ Mean Proportions of Errors (with $S D$ s) for Target/Distractor Pairs } \\
\hline & & & & & 180/320 Grit & 180/180 Grit & $180 / 80$ Grit & $240 / 80$ Grit \\
\hline \multicolumn{9}{|c|}{ Same Hand } \\
\hline 1 & Right index & Right thumb & 14 & 1 & $0.13(0.10)$ & $0.06(0.08)$ & $0.07(0.08)$ & $0.16(0.14)$ \\
\hline 2 & Right thumb & Right index & 16 & 1 & $0.19(0.12)$ & $0.01(0.03)$ & $0.12(0.09)$ & $0.16(0.19)$ \\
\hline \multicolumn{9}{|c|}{ Different Hands } \\
\hline 3 & Right index & Left thumb & 15 & 2 & $0.11(0.12)$ & $0.12(0.09)$ & $0.09(0.10)$ & $0.23(0.33)$ \\
\hline 4 & Right thumb & Left index & 13 & 2 & $0.26(0.09)$ & $0.13(0.11)$ & $0.20(0.13)$ & $0.18(0.23)$ \\
\hline 5 & Left index & Right thumb & 15 & 2 & $0.12(0.11)$ & $0.06(0.10)$ & $0.10(0.13)$ & $0.10(0.13)$ \\
\hline 6 & Left thumb & Right index & 17 & 0 & $0.13(0.12)$ & $0.02(0.04)$ & $0.11(0.10)$ & $0.15(0.19)$ \\
\hline
\end{tabular}

The numbers of participants with errors greater or less than 2.5 SDs from their group mean or with performance at ceiling levels are shown in the column labeled "Excluded $N$." The final set of four columns show the mean proportions of errors and the corresponding SDs for each group of participants. 
Fig. 1 Stimuli and trial sequence. The images in panel (a) show the two surfaces of a single stimulation card. The trial sequence, from first contact to the end of contact with the textures, is shown in panel (b). The perspective in the images is from the left side of the participant. The target digit touched one surface of the card, while the distractor digit simultaneously touched the other surface of the card. The participant is here shown touching the two surfaces using a single hand; however, a similar arrangement was used when the digits were on different hands. a

b Experimenter Participant

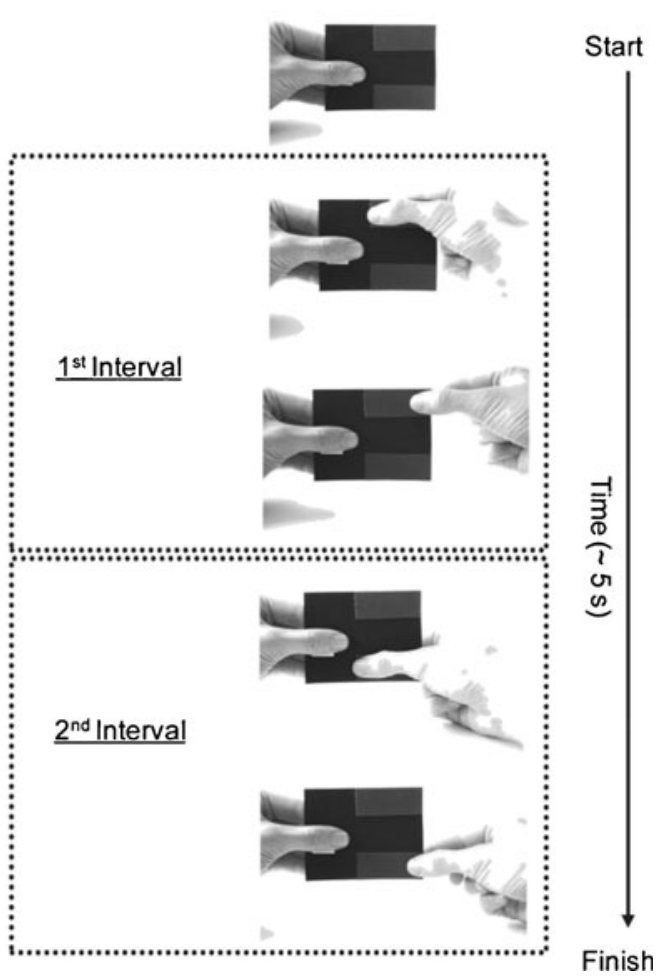

detect and report the interval containing the rough distractor surface. Such behavior would give rise to data with fewer errors in the rough-distractor condition, for reasons unrelated to interference between texture signals on the target and distractor digits. The use of this strategy was circumvented by including the ten rough-swapped trials, ensuring that when the highly salient rough distractor was touched, the very salient difference between that surface and its accompanying target was not a cue to the temporal location of the rough target.

In all experimental conditions, a set of stimulus cards was used for three participants before the sandpaper strips were renewed. This was equivalent to renewing each stimulus after 15 trials. A soft artist's paintbrush and water were used to lightly brush each sandpaper strip between the testing of each of the three participants in order to remove any debris.

Procedure The seated participants faced the experimenter and kept their eyes closed throughout testing. Instructions concerning which digits to form into an opposable grip and how to explore the stimulus surfaces were given at the start of the experiment. As is shown in Fig. 1, the hands were always held in postures in which neither hand was positioned above the other. This postural arrangement was selected so as to avoid the advantage to the upper hand demonstrated by Roberts and Humphreys (2010a). The
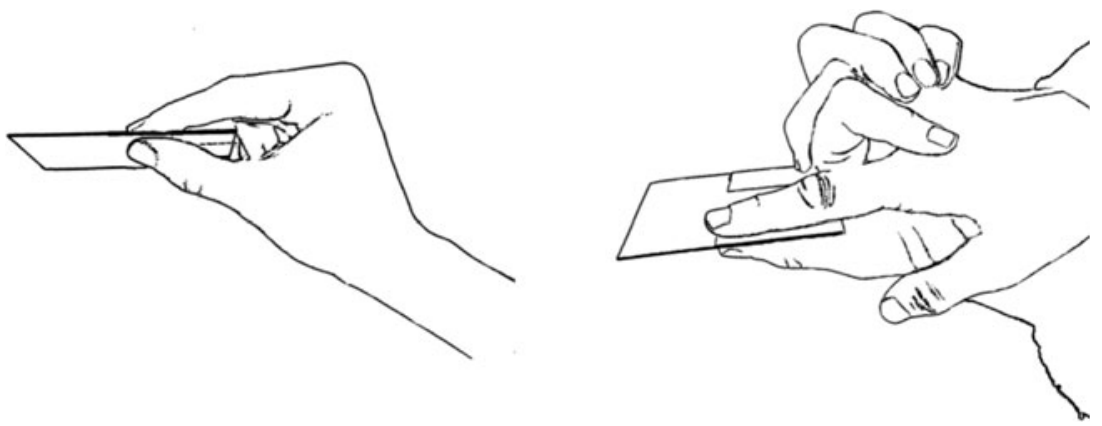

Fig. 2 The within- and between-subjects index-thumb hand postures adopted by different groups of participants. The hands are seen from the participants' visual perspectives (note that participants did not look at their hands, as their eyes were closed during testing). 
participants were instructed to explore the pair of surfaces (the target-and-distractor pair) using one fluid, single sliding movement. This involved applying equal pressure with both digits and minimizing the contact force with the sandpaper surfaces, so that drag resistance was low while ensuring that both digits remained in contact with both surfaces. The experimenter, lightly holding the other end of the stimulus card, used vision and touch to detect any twists and movement arising from unequal forces between the participant's two fingers. This arrangement implies approximately equal normal forces at each digit, although these were not directly measured. Following Roberts and Humphreys (2010a), participants were encouraged to adopt a scanning speed of roughly $25 \mathrm{~mm} / \mathrm{s}$. The experimenter gave verbal feedback to correct any detected deviations from these movement characteristics. The participants were reminded that whilst they were to feel both surfaces simultaneously, they should restrict their attention to the texture at the target digit and ignore the sensations arising from touching the distractor surface with the distractor digit.

Roughness discrimination of the surface touched by the attended digit was measured using a two-interval forced choice paradigm. In the first temporal interval, participants used the digits (the combination of which varied across the different conditions) to touch one target-and-distractor-surface pair. The same digits were then used to touch the other pair of sandpapers on the card. This was the second interval. This sequence can be seen in Fig. 1b. At the end of a trial, participants reported which interval contained the rougher of the two target surfaces. The participants received no feedback about their performance during the experiment. Sound created by contact between the digits and the sandpapers was masked by using white noise played at $52.2 \mathrm{~dB}$ over ear-enclosing noise reduction headphones (Sennheiser PCX 300).

Experimental conditions Sandpaper stimuli were used in this experiment, as such surfaces have often been used to examine roughness perception. More specifically, the present experiment was a continuation of previous work conducted using sandpaper and showing digit interaction effects. Furthermore, sandpaper is a textured surface familiar to many people, with roughness being its prominent feature. However, prolonged exposure to such abrasive surfaces has the capacity to damage the skin and thus alter the physical response to such stimuli. Therefore, this first set of experiments was designed to collect as many observations as possible while limiting contact with the sandpaper surfaces. To this end, each stimulus combination was repeated only ten times, and separate groups of participants performed the task using different combinations of digits.

The 90 participants were divided into six groups, with each group being tested on a different combination of target and distractor digit (see Table 1 and Fig. 1). The experimental factors of interest can be summarized across the groups as follows: For 30 of the participants, the target and distractor digits were on the same hand, and 60 used digits from both hands. The distractor digit was the index finger for 46 participants but the thumb for 44 , and was on the right hand for 47 of the participants and on the left for 43. Details of the combinations of factors for each group are given in Table 1.

On and basis of previous findings that the reports of roughness at an attended digit are biased in the roughness direction of a concurrently stimulated distractor digit (Kahrimanovic et al., 2009; Roberts \& Humphreys, 2010a), the proportion of errors in the present experiment was expected to vary depending on the roughness of the distractor surface: More errors were expected in conditions in which a comparison of the two target surfaces would result in a different response than if the target surface of the standard stimulus was compared with the distractor surface from the comparison stimulus. For example, a trial on which a standard stimulus with a 240/240 (target/distractor) grit pairing was compared with a comparison stimulus of $180 / 80$ grit would be expected to produce fewer errors than would a $180 / 320$-grit pairing, even though the target surfaces being compared ( 240 vs. 180 grit) would be the same in both cases. If the participants performed the task by erroneously attending to distractor surfaces or if the distractor biased the perceived roughness of its accompanying target, there should be a greater tendency to report the standard target as being smoother in the former than in the latter case. Therefore, more errors were anticipated with smooth and with rough-swapped distractors than with either rough or equal distractor surfaces. Following a similar logic, errors were not expected to differ significantly between the rough and equal distractors conditions. The effect of selective attention on texture perception, and in particular any interaction between attentional directions and the identity of the digits and hands exploring the surfaces, has not been widely studied. Perceived roughness has not been found to differ between the thumb and index finger (Verrillo et al., 1999) or between the left and right hands (Lederman, Jones, \& Segalowitz, 1984; Nefs, Kappers, \& Koenderink, 2005). Therefore, there were no a priori expectations about the effect of finger identity or hand dominance on any interdigit interactions found.

\section{Results}

The proportion of errors made in identifying the rougher of the two target surfaces was calculated for each of the distractor surfaces. The data of three participants were removed because they made no errors in three of the four roughness conditions. The data of a further five participants 
were removed because they had errors more than 2.5 times greater than the mean in any of the roughness conditions. The numbers of the excluded participants in different groups are shown in Table 1, along with the mean proportions of errors and the standard deviations for that group.

The data were analyzed using a mixed-design analysis of variance (ANOVA) with Distractor Roughness (rough, smooth, equal, rough-swapped) as the within-subjects factor and Hand (same, different) and Distractor Digit (thumb, index finger) as the between-subjects factors. Graphs of these data can be seen in Fig. 3. Although every trial in the experiment involved selecting the rougher of two surfaces, a 180- or a 240-grit surface, the proportions of errors made by participants varied with the roughness of the distractor surface, $F(3,234)=9.491, p<.001$. No main effects emerged of the number of hands, $F(1,78)=0.660$, $p=.419$, or the particular digit used to feel the distractor surface, $F(1,78)=0.962, p=.330$. More errors occurred with smooth, rough, and rough-swapped distractors than when the distractors were equal in roughness to their respective target surfaces, all $t \mathrm{~s}(81)>3.497, p \mathrm{~s}<.01$. Furthermore, as

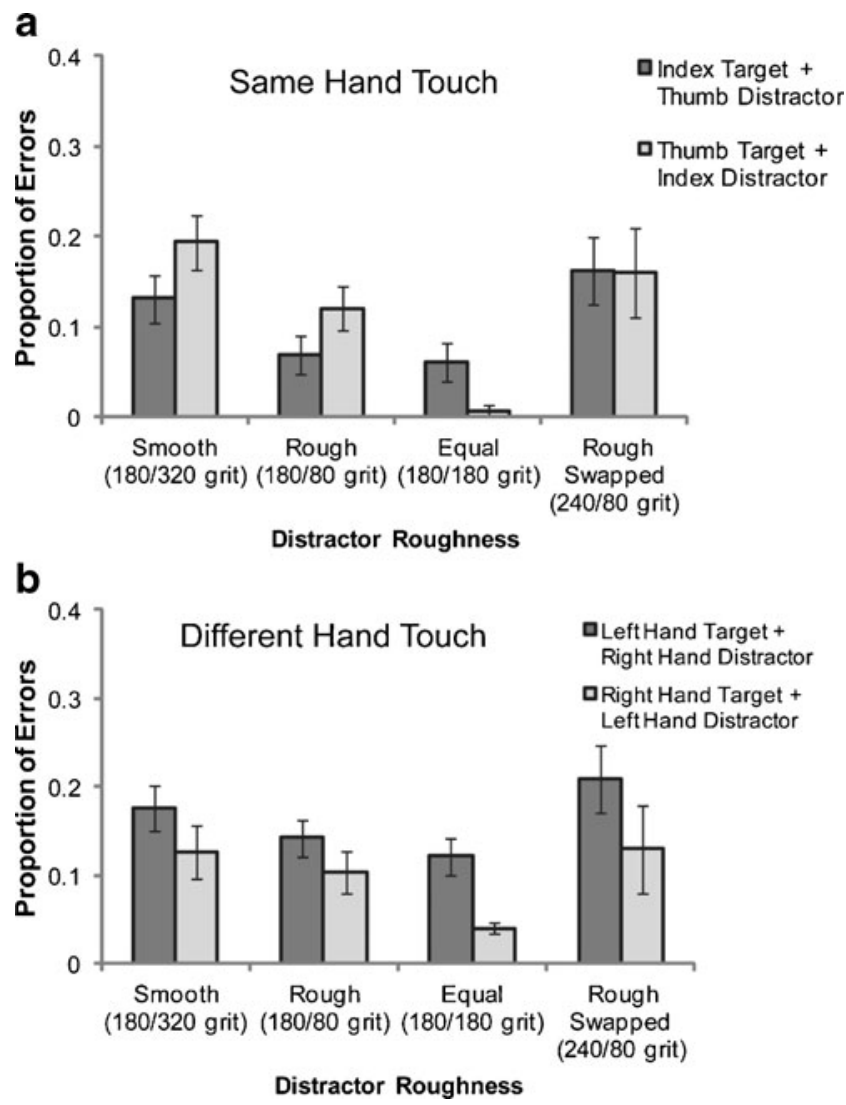

Fig. 3 Proportions of errors produced to the same pair of target stimuli, as a function of distractor roughness. The upper graph, panel (a), shows performance with target and distractor digits on the same hand. The lower graph, panel (b), shows performance when the target and distractor digits were on different hands. Error bars represent one standard error of the mean predicted, identifying the rougher of the pair of target stimuli was more difficult with smooth than with rough distractors, $t(81)=2.534, p=.013$. A trend was also apparent for more errors to occur with rough-swapped than with rough distractors, $t(81)=1.897, p=.061$. No significant interactions were found between the main factors, $F_{\mathbf{S}}<0.598$, all $p \mathrm{~s}>.617$. With one exception, a trend toward an interaction between distractor roughness and digit, $F(3,234)=$ $2.168, p=.092$, reflected a tendency for more errors to occur when using the index finger than when using the thumb with both smooth and rough distractor surfaces, but little difference in performance emerged between the digits with equal or rough-swapped distractors.

Performance was measured with both left- and with righthand distractors in the different-hand condition, but only with right-hand distractors in the same-hand condition. Thus, in the previous analysis combining the same- and different-hand conditions, the data in the different-hand conditions were collapsed over the hand to which the distractor was presented. Little evidence was apparent of differing roughness sensitivity across the hands (Lederman et al., 1984; Nefs et al., 2005). However, an attentional bias toward the dominant hand could possibly have been present - as has been reported for bimanual movement tasks (Buckingham \& Carey, 2009; Peters, 1981; Rabbitt, 1978) - and therefore a greater effect of distractors felt by the right hand. This possibility was explored in an additional ANOVA of the different-hand data. These data, shown in Fig. 3b, were analyzed using a mixed-design ANOVA with Distractor Roughness as the within-subjects factor and Distractor Hand Identity (left, right) and Distractor Digit (thumb, index finger) as the between-subjects factors. As in the overall data, the number of errors produced while discriminating between the target surfaces varied with distractor grit, $F(3,150)=3.345, p=.021$. In addition, more errors occurred with left-hand than with right-hand distractor digits, $F(1,50)=$ $9.314, p=.004$. No other main effects appeared, nor any significant interactions, $F_{\mathbf{S}}<1.951$, all $p \mathrm{~s}>.170$.

\section{Discussion}

The participants taking part in Experiment 1 found it difficult to restrict their attention to a single digit when judging surface roughness. When a pair of adjacent digits on one hand touched different textures simultaneously, reports of the roughness at one of the digits were influenced by the roughness of the surface touched by the other digit. The rougher of a pair of target sandpaper surfaces (touched in sequence) was more likely to be misjudged as being smoother when it was touched simultaneously with a smooth rather than with a rough or an equal-roughness distractor surface on another digit. Interestingly, this pattern of results was not restricted to pairs of digits on the same 
hand; a similar effect of distractor texture was observed when the digits touching the target and distractor surfaces were on different hands.

In addition to being affected by the roughness signals from the distractor digit, the discrimination of roughness at a target digit also depended on the hand to which the accompanying distractor digit belonged. Distractor surfaces touched by left-hand digits were associated with more errors than were those touched by right-hand digits. This increase in errors was present for all three levels of distractor roughness, suggesting that this hand identity effect arose from different processes than were involved in generating the roughness-varying interference between the digits. All of the participants tested were right-handed, and the one-hand conditions were not tested using the left hand. Therefore, the hand identity effects found here may be specific to the left hand, in which case we might see a similar pattern in lefthanders. Alternatively, the left-hand effect may reflect an enhanced distractor effect in, or greater sensitivity of, the nondominant as compared with the dominant hand.

It seems unlikely that the error increase would be due to differing levels of roughness sensitivity on the left versus right or on dominant versus nondominant hands. The ability to judge the roughness of sandpaper and square-wave gratings has previously been found to be unaffected by whether those surfaces were touched using the left or the right hand (Lederman et al., 1984; Nefs et al., 2005). Moreover, the possibility of an attentional bias toward the nondominant left hand also seems unlikely, given that hand-based attentional biases have usually been reported for the dominant hand. Attention has been found to be biased toward the right hand of right-hand-dominant participants during bimanual reaching (Buckingham \& Carey, 2009) and bimanual polyrhythm tapping (Peters, 1981). An increased Simon effect ${ }^{1}$ (Rubichi \& Nicoletti, 2006) has also been observed for responses with the dominant as compared with the nondominant hand. Furthermore, choice reaction times, when the response hand is unknown in advance, are faster when the response is made with the dominant rather than the nondominant hand (Rabbitt, 1978). In all of these cases, when there was either a choice of movement between the two hands or movement of both hands, spatial attention has been biased toward the dominant hand. However, it is possible that touch represents an exception, in which selection is biased in favor of the nondominant hand in right-handers. It would be interesting in future work to explore whether

\footnotetext{
${ }^{1}$ The Simon effect describes situations in which participants make a choice response to a nonspatial attribute of a stimulus (e.g., to its color or form). Responses are made using devices situated on the left and the right. Both accuracy and response times are improved when the irrelevant stimulus location corresponds with the location of the appropriate response device, as compared with cases in which the stimulus and response locations do not match.
}

this bias is specific to roughness perception or is found for other aspects of somatosensation. Furthermore, future contrasts between roughness perception using left-hand digits only as compared with right-hand digits only would make it possible to determine whether the left-hand bias observed in the present experiment is a bias toward the left or toward the nondominant hand.

The main finding of interest here was that roughness judgments at cued digits were affected by the roughness of the surfaces simultaneously touched by digits both on the same and on different hands. Differing effects of rough and smooth distractors on roughness discrimination suggests that the effects of a distractor digit on a cued digit were not due to a nonspecific impairment arising from the presence of roughness signals at a distractor location. It has previously been suggested that same-hand digit interactions arise from the biasing of perceived roughness at an attended digit in the roughness direction of the distractor surface (Kahrimanovic et al., 2009; Roberts \& Humphreys, 2010a), possibly through a weighted integration of the roughness signals at the two hands. In the present experiment, integration of the two sets of sensory signals, one from each digit, would lead to more errors with smooth and with rough-swapped distractors than with distractor surfaces that are equal in roughness to their corresponding attended target surfaces. Following the roughness-biasing argument, a smooth (320-grit) distractor would bias its accompanying target (180 grit) to feel smoother than would be the case with a 180-grit distractor. This would increase the difficulty in deciding whether the 180-grit target is rougher than a 240-grit target. A similar logic applies in the rough-swapped condition. This pattern of responses was found in Experiment 1. However, the biasing account of multidigit roughness would also predict few errors in the rough-distractor condition (180/80-grit target/distractor pair). The pairing of a rough target with a rough distractor should have resulted in an increase in the perceived roughness of the target, making it more distinct from the smoother (240-grit) target with which it would be compared. In contrast to this prediction, significantly more errors occurred with rough than with equal-roughness distractors. An alternative account for the interactions observed between the digits may lie in the relative salience of the different distractors: More-salient distractor surfaces might exert a greater attentional pull than would other, less-salient surfaces. This might lead to increased difficulty judging the roughness of the accompanying surface textures. However, against this account, the greatest proportion of errors were observed with the smooth distractor (320 grit). This surface has previously been judged as relatively less salient than the rough, 80-grit surface (see Roberts \& Humphreys, 2010a). Furthermore, the effect of the rough distractor was not the same in the rough-swapped (240/80-grit target/distractor 
pair) and the rough (180/80-grit target/distractor pair) conditions. Together, the latter pair of results do not favor a salience-based distractor account of the present data. However, the difference between the rough-swapped and rough distractors was not reliably present across the experimental conditions and was accompanied by high levels of variability between participants with rough-swapped distractors. Furthermore, the absence of a pairing of the smooth distractor with the smoother of the target surfaces makes it less certain that the present results did not arise from a salience-dependent attentional pull of the distractor surface.

These questions were addressed in a second experiment. Additional pairs of smooth and rough distractors were used to examine more closely the effect of distractor roughness on texture perception at an attended location. As before, participants judged which of a pair of target surfaces was rougher. However, in Experiment 2, the target surfaces were more closely matched (220 and 240 grit, as compared with 180 and 240 grit in the earlier experiment), in order to avoid any possible ceiling effects. Furthermore, each target surface was paired with each distractor surface. A saliencedependent effect of distractor roughness would lead to performance that varied with the roughness of the distractor surfaces but did not change systematically when the distractor was paired with a rough or smooth target surface. Consistent changes in the effect of distractor surfaces on smooth as compared with rough targets would be more indicative of perceptual biasing caused by the distractor.

\section{Experiment 2}

\section{Method}

Participants A group of 13 volunteers between the ages of 18 and 23 (median 19) years took part in this experiment; ten were female and three male. All of the participants selfreported as being right-handed, had normal or corrected-tonormal vision, and gave informed consent before participating. Each participant received $£ 10$ sterling or the equivalent in undergraduate course credits for their participation.

Stimuli The sandpaper grit range was extended from that used in Experiment 1, in order to include textures differing more subtly in roughness from each other. The target pair selected for Experiment 2 had grits (and mean particle sizes, in micrometers) of $220(60)$ and 240 (50). This smaller difference in roughness, as compared with the targets used in Experiment 1, was expected to lead to an increase in error rates as compared with those found in Experiment 1. The distractor range comprised distractors close in roughness to the target surfaces-180 (80) and 320 (40) grit-and distractors farther in grit value from the target pair, with grits of 80 (192) and 600 (15).

On a trial, the target/distractor pairing of one of the two stimulus intervals consisted of a pair of surfaces with different grit levels. The target-and-distractor pair in the other stimulus interval were surfaces with equal grit levels. On half of the trials, the rough target surface (220 grit) differed from its accompanying distractor, and the smooth target differed from its distractor on the remaining trials. Each target surface was presented with each of the distractor surfaces eight times.

The stimuli and procedure employed in Experiment 2 were the same as those described for Experiment 1 in all other respects.

Experimental conditions Participants always used their right thumb to explore the distractor surface. The right index finger was used to feel the distractor surface on half of the trials (the same-hand condition), and the left index finger touched the distractor surface on the remaining trials (different-hand condition). Each hand condition was tested in two blocks of trials, with the two conditions run in an ABBA design.

Data analysis The proportions of errors were calculated for each condition. These data can be seen in Fig. 4. The upper part of the figure, panel (a), shows the proportions of errors made when the distractor and target digits belonged to the same hand. The lower part of Fig. 4 shows performance when the two digits were from different hands. The data from three participants were excluded from further analyses, as these participants produced errors more than two standard deviations from the group mean with three or more different distractor surfaces.

\section{Results}

The data were analyzed using a repeated measures ANOVA with Hand (same, different), Target With Unequal Distractor (rough target, smooth target), and Distractor Roughness (80, 180,320 , and 600 grit) as factors. More errors were produced when the rougher than when the smoother target surface was paired with distractors of unequal roughness, $F(1,9)=26.621$, $p<.01$. The significant interaction between this factor and distractor roughness, $F(3,27)=35.767, p<.01$, arose from the opposite effects of distractor roughness on the pair of target stimuli. No other main effects or interactions were apparent, all $F_{\mathrm{S}}<1.310$, all $p \mathrm{~s}>.292$. Paired-samples $t$ tests comparing the errors with smooth and rough targets were carried out for each distractor in both the same- and different-hand conditions. Significantly fewer errors occurred with smooth distractors (600 and 320 grit) when they were paired with the smoother rather than the rougher of the target surfaces, all $t \mathrm{~s}(9)>2.501$, all $p \mathrm{~s}<.05$. In contrast, roughness 


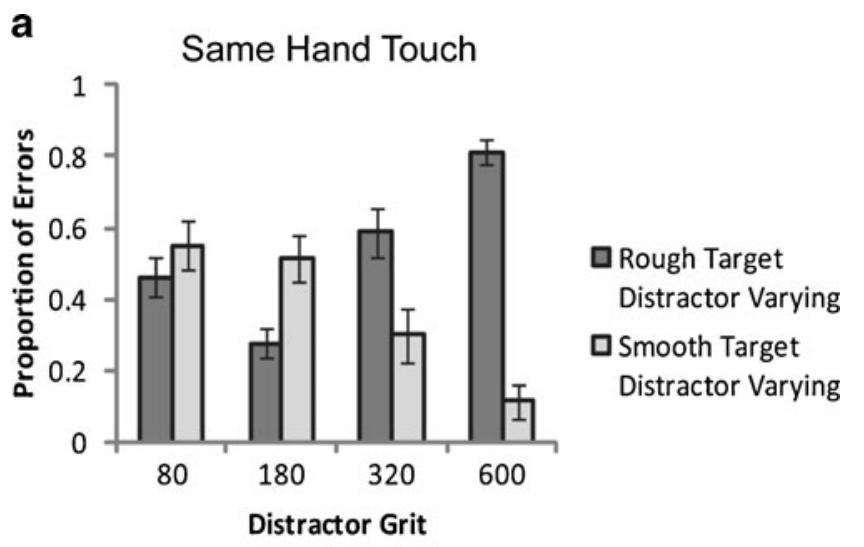

b

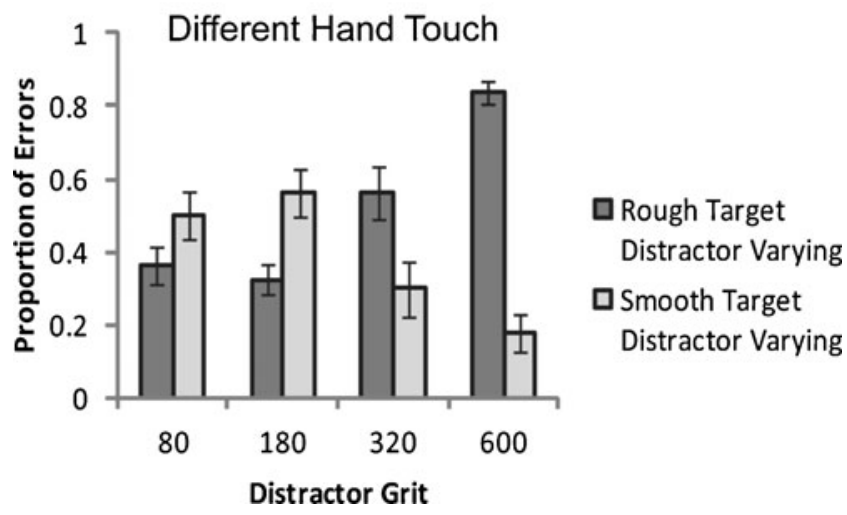

Fig. 4 Proportions of errors produced to target stimuli, as a function of distractor roughness. Errors when the varying distractors were paired with the smoother of the target surfaces are shown in light gray, and errors when distractors were paired with the rougher of the target surfaces are shown in dark gray. The upper graph, panel (a), shows performance with target and distractor digits on the same hand. The data when the target and distractor digits were from different hands are shown in panel (b). Error bars represent one standard error of the mean

discrimination was worse when the 180-grit rough distractor was paired with the smoother than with the rougher target surface: $t(9)=-3.143, p=.012$, for the same-hand condition, and $t(9)=-3.143, p=.012$, for the different-hand conditions. Interestingly, more errors occurred with 80-grit distractors paired with the smoother rather than the rougher target. However, this difference was small for the different-hand condition, $t(9)=-2.538, p<.032$, and not present in the same-hand condition, $t(9)=-0.843, p=.421$.

Measures taken when the distractors were no different from their targets were not included in the main analysis. In these conditions, a trend emerged toward better roughness discrimination with target and distractor digits on the same hand rather than different hands, $t(9)=-2.012, p=.075$.

\section{Discussion}

In Experiment 2, the effect of distractor surfaces varied with the relative roughness of the accompanying target surface.
Pairs of target surfaces, touched in sequence, were easier to discriminate when the smoother of the targets was paired with a smooth rather than a rough distractor surface. A complementary effect was found when the rougher of the targets was paired with a rough distractor surface. This distractordependent improvement was found for target/distractor pairs touched by digits from the same hand, and also for exploration using digits from different hands. The use of highly similar target surfaces, discriminable at around chance levels in the presence of equal distractors, makes it unlikely that the distractor surfaces had a different effect on the perceived roughness of the rougher of the target surfaces than on the smoother target surface. The pattern of behavior is better explained by an interaction between integration of the roughness signals on the two digits and the two-interval forced choice design of the discrimination task.

Although behavior consistent with the integration of the roughness signals in the two digits was apparent for surfaces of 180 grit and smoother, it was less evident for the relatively rougher 80 -grit surface. It is possible that the high salience of the 80-grit surface may have disrupted perception of the target surfaces either by masking simultaneously touched surfaces or drawing attention from them. Alternatively, altered interactions between the digits with 80-grit as compared with smoother-grit distractors may reflect fundamental processing differences between groups of rough and smoother surfaces. There is psychophysical evidence that different sensory processes are engaged by what are labeled coarse (rough) and fine (smoother) surfaces (Hollins, Bensmaia, \& Washburn, 2001; Hollins \& Risner, 2000). The duplex theory of texture perception holds that coarse textures are mediated by spatial codes using the spatial distribution of forces across the skin when the skin and surface come into contact. In contrast, fine surfaces are mediated by vibration codes set up by the movement of the skin relative to the textured surface. Consistent with this dual-processing model, the discrimination of fine sandpapers (average particle sizes $<100 \mu \mathrm{m}$ ) has been found to be impaired in static as compared with dynamic contact conditions, whereas the discrimination of coarse surfaces (average particle sizes $>100 \mu \mathrm{m}$ ) was relatively unaffected (Hollins \& Risner, 2000). The 80-grit distractor used in the present experiments falls within the spatially coded range, whereas the remaining textures are within the vibration-coded range. It is possible that integration only occurs between textures coded in a similar manner, or that integration of roughness signals between the digits only occurs with fine surfaces.

\section{General discussion}

The close proximity of the digits on the body and relatively limited scope for spatial separation, coupled with the tendency 
to produce in-phase movements of the digits during textural exploration, make it likely that the digits on a single hand often transmit highly correlated textural information when touching surfaces. The same is less likely to be true for a pair of digits on different hands that are, ostensibly, free to move independently of each other. This freedom reduces the potential correlation in sensory signals on the two hands. Thus, there is reason to expect the integration of texture signals within but not between the hands. Previous studies have shown behavior indicative of integration of the roughness signals from within the hand. Roughness judgments for the surfaces touched by a cued digit were shifted in the roughness direction of the surface touched by a neighboring distractor digit on the same hand (Kahrimanovic et al., 2009; Roberts \& Humphreys, 2010a). That finding was supported by the results of the present study, in which, when a pair of adjacent digits on a hand touched different textures simultaneously, reports of the roughness at one of the digits were influenced by the roughness of the surface touched by the other digit. A novel finding here was that, contrary to the report of Dorsch et al. (2001), the interfering effect of distractor roughness was not restricted to digits on the same hand. A similar effect of a distractor texture was observed when the cued and distractor digits were on different hands. Interestingly, sensory interactions between surfaces touched by pairs of digits were altered with the use of very rough surfaces. The effect of this rough surface was more indicative of an impairment to texture processing than of the swaying of roughness perception in one direction or another.

The failure to filter out roughness signals from concurrently stimulated digits may reflect the automatic processing of roughness signals across the body. Haptic search tasks provide evidence for the parallel processing of texture signals across the hands (Lederman et al., 1984; Lederman \& Klatzky, 1987; Plaisier, Bergmann Tiest, \& Kappers, 2008). In those experiments, participants are typically asked to report whether a target property is present amongst varying numbers of distractor stimuli of the same perceptual dimension. ${ }^{2}$ For example, participants touching a set of textured surfaces with six of their fingers would make a speeded decision regarding whether one of the digits touched a rough surface or whether the surfaces under all of the fingers were smooth. The time taken to find target properties is informative of the processing required for perception of that dimension. Increases in response times as the number of fingers stimulated increases suggest that additional, serial processing steps are needed for each digit or that there is a distribution of limited processing

\footnotetext{
${ }^{2}$ Lederman and Klatzky (1997) defined a dimension as a domain of variation that is accessible to the perceptual system. Examples of continuous dimensions include roughness and compliance, whereas binary dimensions might include surfaces with a feature oriented to the left or the right. Properties are defined as single values in consciously perceptible domains.
}

capacity across more digits. On the other hand, the fact that response times are unchanged by the number of digits being searched suggests that no additional processing costs are associated with each extra digit. Generally, texture search experiments have found that the time taken to search textured distractors for a surface of a target roughness is relatively unaffected by the number of fingers to be searched (Lederman et al., 1984; Lederman \& Klatzky, 1987; Plaisier et al., 2008). This is in contrast to haptic search tasks based on the spatial features of tactile stimuli (e.g., discriminating whether a left-oriented bar is present amongst a series of right-oriented bars), in which target detection times increase significantly with the number of fingers to be searched (Lederman \& Klatzky, 1997; Overvliet, Smeets, \& Brenner, 2007). Lederman and Klatzky (1997) suggested that although searches of these spatially coded properties involve the serial processing of signals from the fingers, material tactile dimensions such as texture and compliance are processed in parallel across the fingers. Furthermore, the hand and finger movements made during unrestrained haptic search show a pattern consistent with the latter suggestion. Plaisier et al. allowed their participants to freely explore a flat surface containing between three and 11 textured patches. The participants' task was to search for a patch with the target texture amongst patches with distractor textures. Rather than moving each finger individually, participants kept their hands flat, their fingers together, and explored the surface with sweeps of the whole hand. Deviations from this pattern occurred under increased processing demands - that is, when the task became more difficult. Under these conditions, participants switched to single-point exploration patterns.

However, it appears that a cost is associated with the ability to process texture in parallel across multiple digits. Under some conditions (those explored here and previously: Kahrimanovic et al., 2009; Roberts \& Humphreys, 2010a), in which participants are prevented (usually by instruction) from withdrawing some contact points from surfaces, it is difficult to prevent the processing of textures at task-irrelevant digits. Most interestingly, the signals from these digits systematically affect texture perception at an attended location in a manner consistent with some form of integration across the digits. Thermal perception when touching with multiple digits has similarly been found to show perceptual interactions across the digits (Ho, Watanabe, Ando, \& Kashino, 2011). A thermally neutral surface touched by one digit was perceived as being equally warm (or cold) as a pair of heated (or cooled) flanking surfaces touched simultaneously by adjacent digits. However, in contrast to the present findings for roughness, no evidence emerged of thermal referral when the digits contacting the temperature-controlled surfaces were on different hands (Ho, Watanabe, Ando, \& Kashino, 2010). Referred changes in temperature sensation were most likely to occur when the three digits contacting surfaces were adjacent and on the same hand. Interactions 
between the digits diminished when the digits were on the same hand but not adjacent, and were least evident when one of the digits was on another hand. Although roughness and thermal qualities are both material aspects of surfaces, this difference in the findings for the two submodalities may reflect the recruitment of different processes for the perception of an object's roughness as compared with its temperature. Alternatively, the difference in findings between the experiments may reflect other stimulus differences. In the study by Ho et al. (2010), the thermal stimuli on the two hands were felt by touching stimulus pads on two clearly separate objects. Knowledge about whether tactile stimuli originate from a single as compared with two objects has been shown to change the distribution of tactile attention (Gillmeister, Adler, \& Forster, 2010): It is harder to withdraw attention from a distant (in both spatial and somatotopic coordinates) body part when it contacts the same object as an attended body part, as compared with when the two body parts touch separate objects. It is not clear whether this factor of Same/Separate Object played a role in Ho et al.'s (2010) failure to show an interaction between the digits in the bilateral-touch conditions. This factor may also have played a role in generating the bilateral interference observed in the present data. Here, the pair of sandpaper surfaces (cued and distractor surfaces) in each stimulus interval were attached to either side of the same card. The combination of these surfaces and the card produced a single stimulus that was less than $2 \mathrm{~mm}$ thick. It remains to be seen whether similar distractor interaction with texture judgments would be observed across the hands when the digits of the two hands touch clearly different objects.

The spatial separation of somatosensory stimuli is another factor that has been shown to influence perception during bilateral touch. Studies of tactile attention have shown an influence from stimuli on one side of the body on the perception of tactile stimuli presented on the opposite side. The identification of vibrotactile stimuli applied to the digit of a hand was slower and more error-prone when dissimilar vibrotactile stimuli were applied to the homologous digit on the opposite hand than when a distractor was not present (Driver \& Grossenbacher, 1996; Roberts \& Humphreys, 2010b). Similar results have been found in tasks in which the spatial elevation of the target vibrotactile stimuli was reported (Soto-Faraco et al., 2004). Interestingly, the interference between vibrotactile stimuli on the two hands was greatest when the pair of stimulated body parts were held spatially close to each other rather than far apart. This finding has been interpreted as evidence that attention in the somatosensory system can operate within nonbodily coordinates, either drawn by or shared with the stimulus on an unattended hand, depending on the relative separation of the two hands. Further support for this idea has come from work by Lakatos and Shepard (1997) showing that attention is more rapidly moved between pairs of locations on the body when those locations are held close in space, as compared with far apart. Difficulty ignoring spatially close tactile stimuli may not be specific to vibration, but may apply to other tactile properties such as surface roughness. At present, the role of spatial proximity in the bilateral perception of roughness is unclear. Work investigating whether the influence of a distractor surface is changed with spatial separation from a target surface is currently underway.

Given the relative flexibility with which we are able to use our two hands in coordinated exploration, it is interesting to examine how somatosensory information is processed across the body. The present results suggest that interactions between roughness signals from the digits are not restricted to those signals originating from neighboring regions of the body. Instead, behavior consistent with the integration of sensory signals was observed when rough textures were touched by digits distant on the body but close in space. Furthermore, some evidence has indicated that the interaction between surfaces touched by different digits is constrained by whether those surfaces fall within roughness boundaries thought to involve similar sensory processes. However, it remains to be seen whether the behavior observed in the present experiment would occur if only coarse surfaces were touched, whether it would occur for all digit combinations across the hands or is specific to combined thumb-and-forefinger use, whether similar behavior could be found in single-hand exploration with the nondominant hand, and whether similar integration mechanisms would be involved with different degrees of spatial separation between the exploring digits. Furthermore, the precise nature and neural bases of the interactions between roughness signals from digits within and between hands remains to be elucidated.

Author Note The author was funded by a British Academy Fellowship.

\section{References}

Buckingham, G., \& Carey, D. P. (2009). Rightward biases during bimanual reaching. Experimental Brain Research, 194, 197-206.

Dorsch, A. K., Hsiao, S. S., Johnson, K. O., \& Yoshioka, T. (2001). Tactile attention: Subjective magnitude estimates of roughness using one or two fingers. In Neuroscience 2001 Abstracts. Washington, DC: Society for Neuroscience.

Driver, J., \& Grossenbacher, P. G. (1996). Multimodal spatial constraints on tactile selective attention. In T. Inui \& J. L. McClelland (Eds.), Attention and performance XVI: Information integration in perception and communication (pp. 209-235). Cambridge MA: MIT Press.

Ekman, G., Hosman, J., \& Lindstrom, B. (1965). Roughness, smoothness, and preference - A study of quantitative relations in individual subjects. Journal of Experimental Psychology, 70, 18-26.

Gillmeister, H., Adler, J., \& Forster, B. (2010). Object-guided spatial attention in touch: Holding the same object with both hands delays attentional selection. Journal of Cognitive Neuroscience, 22, 931-942. 
Ho, H.-N., Watanabe, J., Ando, H., \& Kashino, M. (2010). Somatotopic or spatiotopic? Frame of reference for localizing thermal sensations under thermo-tactile interactions. Attention, Perception, \& Psychophysics, 72, 1666-1675. doi:10.3758/ APP.72.6.1666

Ho, H.-N., Watanabe, J., Ando, H., \& Kashino, M. (2011). Mechanisms underlying referral of thermal sensations to sites of tactile stimulation. Journal of Neuroscience, 31, 208-213. doi:10.1523/JNEUROSCI.2640-10.2011

Hollins, M., Bensmaia, S. J., \& Washburn, S. (2001). Vibrotactile adaptation impairs discrimination of fine, but not coarse, textures. Somatosensory and Motor Research, 18, 253-262.

Hollins, M., \& Risner, S. R. (2000). Evidence for the duplex theory of tactile texture perception. Perception \& Psychophysics, 62, 695705 .

Kahrimanovic, M., Bergmann Tiest, W. M., \& Kappers, A. M. (2009). Context effects in haptic perception of roughness. Experimental Brain Research, 194, 287-297.

Lakatos, S., \& Shepard, R. N. (1997). Time-distance relations in shifting attention between locations on one's body. Perception \& Psychophysics, 59, 557-566.

Lederman, S. J., Jones, B., \& Segalowitz, S. J. (1984). Lateral symmetry in the tactual perception of roughness. Canadian Journal of Psychology, 38, 599-609.

Lederman, S. J., \& Klatzky, R. L. (1987). Hand movements: A window into haptic object recognition. Cognitive Psychology, 19, 342368.

Lederman, S. J., \& Klatzky, R. L. (1997). Relative availability of surface and object properties during early haptic processing. Journal of Experimental. Psychology: Human Perception and Performance, 23, 1680-1707. doi:10.1037/0096-1523.23.6.1680
Nefs, H. T., Kappers, A. M. L., \& Koenderink, J. J. (2005). Intermanual and intramanual tactual grating discrimination. Experimental Brain Research, 163, 123-127.

Overvliet, K. E., Smeets, J. B. J., \& Brenner, E. (2007). Parallel and serial search in haptics. Perception \& Psychophysics, 69, 10591069. doi:10.3758/BF03193944

Peters, M. (1981). Attentional asymmetries during concurrent bimanual performance. Quarterly Journal of Experimental Psychology, $33 A, 95-103$.

Plaisier, M. A., Bergmann Tiest, W. M., \& Kappers, A. M. L. (2008). Haptic pop-out in a hand sweep. Acta Psychologica, 128, 368 377. doi:10.1016/j.actpsy.2008.03.011

Rabbitt, P. M. (1978). Hand dominance, attention, and choice between responses. Quarterly Journal of Experimental Psychology, 30, 407-416.

Roberts, R. D., \& Humphreys, G. W. (2010a). The role of somatotopy and body posture in the integration of texture across the fingers. Psychological Science, 21, 476-483.

Roberts, R. D., \& Humphreys, G. W. (2010b). Visual context and practice change the distribution of attention in touch. Brain Research, 1351, 185-197.

Rubichi, S., \& Nicoletti, R. (2006). The Simon effect and handedness: Evidence for a dominant-hand attentional bias in spatial coding. Perception \& Psychophysics, 68, 1059-1069. doi:10.3758/ BF03193709

Soto-Faraco, S., Ronald, A., \& Spence, C. (2004). Tactile selective attention and body posture: assessing the multisensory contributions of vision and proprioception. Perception \& Psychophysics, 66, 1077-1094.

Verrillo, R. T., Bolanowski, S. J., \& McGlone, F. P. (1999). Subjective magnitude of tactile roughness. Somatosensory and Motor Research, 16, 352-360. 